\title{
Redaktionelle Richtlinien
}

\section{Allgemein}

Das Forstwissenschaftliche Centralblatt- Tharandter Forstliches Jahrbuch als Zeitschrift für das gesamte Forstwesen, für Umweltforschung und Naturschutz widmet sich der Darstellung und der Vermitrlung wissenschaftlicher Arbeit und ihrer Ergebnisse. Neben den klassischen Inhalten forstwissenschaftlicher Forschung werden verstärkt Umweltthemen und Fragen des Arten- und Biotopschutzes behandelt, die über das Beziehungsgefüge Wald hinausgreifen. Im Sinne diescr Thematik versteht sich das Forstwissenschaftliche Centralblatt - Tharandter Forstliches Jahrbuch als ein Forum für die Behandlung wichtiger, häufig kontrovers diskutierter Fragestellungen.

Die Veröffentlichung erfolge in deutscher oder englischer Sprache. Persönliche Würdigungen und Tagungsberichte werden nicht aufgenommen.

\section{Einreichung und Annahme von Manuskripten}

Bitte reichen Sie alle Manuskripte ein bei:

Professor Dr. H. Pretzsch

Lehrstuhl für Waldwachstumskunde

Forstwissenschaftliche Fakultät

der Technischen Universität München

Am Hochanger 13

D-85354 Freising

Prof. PretzsCH ist Schriftleiter im Sinne des Berliner Pressegesetzes. Er bestätigt die Annahme von Manuskripten oder informiert über ihre Ablehnung. Er ist berechrigt, in den zur Veröffentlichung eingereichten Manuskripten Änderungen geringfügiger Art vorzunehmen und im Einvernehmen mit den Autoren Kürzungen oder wesentliche Änderungen durchzuführen; er entscheidet, in welchem Heft und an welcher Stelle die jeweilige Arbeit veröffentlicht wird. Die Entscheidung über die Annahme triffe das Herausgebergremium. Mitglieder sind die Professoren Dr. D. ECKSTEIN, Hamburg, Dr. S. Fink, Freiburg, Dr. A. Fischer, Freising, Dr. B. v. Lupke, Göttingen, Dr. F. MAkeschin, Tharandt, Dr. H. Pretzsch, Frcising, Dr. H. BugmanN, Zürich, und Dr. J. SPORK, Wien.

Bitte reichen Sic unter Beachtung der Autorenhinweise drei satzreife Exemplare mit Dateien auf Diskette, zip oder CD-ROM ein und behalten Sie eine Kopie Ihres Manuskriptes. Mit der Annahme Ihres Manuskriptes erhalten Sie ein Formular „Manuskripteinreichung“, das binnen 5 Tagen an den Herausgeber zurück zu senden ist. Dieses umfaßr die vollständige Korrespondenzanschrift des Autors, die Rechtsübertragung und die Regelung evtl. Druckkostenzuschüsse.

Sämtliche Beiträge gehen durch ein Reviewsystem und werden dabei von mindestens zwei Gutachtern beurteilt.

Ihr Manuskript wird 3 Monate nach der Veröffentlichung vernichtet. Zurückgesendet werden lediglich gekennzeichnete Unikate. Für die unversehrte Rücksendung bzw. den Postweg übernimmt der Verlag keine Gewähr.

\section{Anforderungen an Manuskripte}

\section{1 Äußere Form}

Das Manuskript besteht aus dem Ausdruck des Textes und einem Verzeichnis aller Bilder und Tabellen mit Bildunterschriften und Tabellenüberschriften auf separatem Blatt. Nach Möglichkeir sind Zeilennummerierungen zu verwenden. $\mathrm{Zu}$ allen Bildern, Tabellen und 
Einträgen im Literaturverzeichnis muß ein Textbezug existieren. Die Zuordnung muß eindeutig sein. Alle Bilder liegen als reprofähige Vorlagen bei (mit Beschriftung: Autor, Kurztitel, Nummer). Evtl. Korrekturwünsche aus dem Begutachtungsverfahren sind komplett in die Dateien eingearbeitet.

Jeder Originalarbeit ist eine deutsche Zusammenfassung möglichst mit englischer Übersetzung (auch des Titels, der Abbildungsunterschriften und Tabellenüberschriften) beizufügen. Der Zusammenfassung und der Summary sind Schlüsselwörter/Key words anzufügen. Die Anschrift des Autors (der Autoren) soll dem Literaturverzeichnis folgen. Datenträger müssen PC Windows-kompatibel sein und dürfen außer den akruellen keine weiteren Dateien enthalten. Ein beigefügtes Dateiverzeichnis enthält den Dateinamen, das Programm und seine Version. Bitte halten Sie Text- und Bilddateien unbedingt getrennt. Der Text sollte in Standardsoftware (Microsoft Word, Word Perfect) oder rtf-Format erstellt sein, ohne Silbentrennprogramme oder manuelles Trennen. Bitte verwenden Sie keine Fußnoten.

\subsection{Umfang}

Der Manuskriptumfang soll bei eineinhalbzeiliger Schreibwcise incl. Abbildungen und Tabellen einen Umfang von 15 Seiten nicht überschreiten.

\subsection{Maßeinheiten, Abkürzungen und Nomenklatur}

Grundsätzlich sind alle Wörter auszuschreiben. So kann zum Beispiel keinesfalls „forstwirtschaftlich" durch "forstw." wiedergegeben werden. Millionen sind abgekürzt mit "Mio." wiederzugeben, für Milliarden wird „Mrd.“ gesetzt. Anstelle von „v. H." wird stets "\%" verwendet.

Alle Größen-, Gewichts- und Temperaturangaben werden entsprechend den geserzlich festgelegten „Einheiten im Mcßwcsen“, Stand 1978, erbeten.

\subsection{Abbildungen und Tabellen}

Wenn Sie Ihre Abbildungen in elektronischer Form einreichen möchten (immer mit Ausdruck), verwenden Sie bitte ein neutrales Datenformat wic TIFF oder EPS. Ungeeignet sind Powerpoint und Word Grafik. Verwenden Sie keine pixelorientierten Programme. Scans (nur als TIFF) sollten eine Auflösung von $300 \mathrm{dpi}$ (Halbton) bzw. 600 bis $1200 \mathrm{dpi}$ (Strichmodus) haben, bezogen auf die Veröffentlichungsgröße. Bitte liefern Sie die Bilddaten in s/w. Wenn Sic Farbabbildungen integrieren möchten, müssen wir einen Druckkostenzuschuß berechnen. Verwenden Sie bitte in diesem Fall den CMYK-Farbraum (Druckfarben), nicht RGB (Bildschirmdarstellung). Eine Bildbearbeitung durch den Verlag ist nur bei Kostenübernahme möglich.

Motiv und Maßstab (Landkarten, mikroskopische Aufnahmen usw.) sollten auf die Veröffentlichungsgröße abyestimmt sein. Gewünschte Bildausschnitte sind auf einer Kopie der Vorlage oder Pergamentpapier zu kennzeichnen. Der Ausdruck sollte per Laserdruck mit maximaler Auflösung (>600 dpi) erfolgen. Farbbilder können (unter möglichem Verlust farblicher Differenzierungen) s/w reproduziert werden. Strichzeichnungen sollten nur als Umrißzeichnungen angefertigt sein und nicht mit Halbton (Foto, Graustufen) vermische sein. Verwenden Sie keine Rasterflächen; grobe Schraffuren sind möglich.

Bitte schließen Sic Graphen (grundsätzlich nur als 2-D Darstellung!) mit $x$ - und y-Achse niche in cinen Rahmen ein. Vergessen Sie nicht Achsenbeschriftungen und Einheiten. Dic Bildunterschrift sollte die Aussage der Gratik möglichst genau wiedergeben und darf im Bild nicht wiederholt scin.

Tabellen sind mit Tabulatoren oder Tabcllentunktion zu erstellen. 


\subsection{Literatur}

Autorennamen sind in Kapitälchen (nicht in Versalien) zu schreiben. Bietet Ihr Textverarbeitungsprogramm Ihnen diese Möglichkeit nicht, schreiben Sie Autorennamen bitte in der üblichen Groß- und Kleinschreibung, und heben Sie sie durch eine Unterstreichung hervor. Wissenschaftliche botanische und zoologische Gattungs-, Art- und Unterartnamen werden kursiv geschricben.

Hinweise auf die zitierte Literatur erfolgen durch Angabe der Verfassernamen mit der Jahreszahl der Veröffentlichung - beide Angaben in Klammern - z. B. (REHFUESS 1990). Bei zwei Autoren werden beide Namen im Text aufgeführt, z. B. (AMMER u. PROBSTL. 1991); bei drei und mehr Autoren wird nur der erste Autor zitiert mit dem Zusatz „et al.", z. B. (FEGER et al. 1991). Falls der Verfasser im Textzusammenhang erwähnt wird, ist nur die Jahreszahl in Klammern anzugeben, z. B. „MAYER und SCHMIDT (1991) berichten ...". Sind im Literaturverzeichnis mehrere Veröffentlichungen eines Autors aus demselben Veröffentlichungsjahr aufgeführt, so sind sie mit a, b, c, usw. zu bezeichnen, z. B. (AMMER 1992 a).

\section{Bedingungen für die Veröffentlichung}

Mit Übersendung des Manuskriptes an den Verlag versichert der Autor, daß sein Beitrag sowie die durch ihn selbst beschafften fremden Text- und Bildvorlagen keine Rechte Dritter verletzen, daß er allein befugt ist, über die daran bestehenden urheberrechtlichen Nutzungsrechte zu verfügen. Der Autor wird sich während der Laufzeit des Vertrages jeder anderweitigen Vervielfältigung und Verbreitung bzw. unkörperlichen Übertragung und Wiedergabe des Beitrages (z. B. im Internet) enthalten. Der Autor überträgt dem Verlag räumlich unbeschränkt für die Dauer des gesetzlichen Urheberrechts das ausschließliche Recht der Vervielfältigung und Verbreitung (Verlagsrecht), und zwar ohne Mengenbegrenzung, das Recht der unkörperlichen Wiedergabe des Werks, das Recht, das Werk jedermann zum individuellen Abruf zugänglich zu machen, sowie das Recht der Lizenzvergabe an Dritte im In- und Ausland zur Ausübung der übertragenen Nebenrechte. Der Verlag ist zur Verwertung der ihm zustehenden Nutzungsrechte befugt, aber nicht verpflichter.

Die Veröffentlichung der Beiträge in der Zeitschrift ist honorarfrei. Die Verfasser von Originalarbeiten erhalten unberechnet 25 Autoren-Sonderdrucke. Erlöse, die durch Vergabe von Nachdruck-, Übersetzungs- oder sonstigen Wiedergaberechten erziclt werden, werden zwischen Autor und Verlag hälftig geteilt.

\section{Korrekturen und zusätzliche Sonderdrucke}

Sie erhalten eine Korrekturfahne ohne Manuskript (wenn Änderungen unterblieben), die umgehend gegengezeichnet werden sollte - bitte stellen Sie Ihre Erreichbarkeit sicher. Die Autorenkorrektur beschränkt sich auf Satzfehler.

Auf einem der Korrekturfahne beiliegenden Formular können Sie zusätzliche Sonderdrucke bestellen. Sofern nicht anders angegeben, werden Sonderdrucke und Belegexemplare an den Korrespondenz- bzw. Erstautor gesandt. Nachträgliche Sonderwünsche sind mit einem Preis-Aufschlag verbunden. 